\title{
COVID-19 associated multisystemic inflammatory syndrome in 614 children with and without overlap with Kawasaki disease-Turk MIS-C study group
}

\author{
Dilek Yilmaz Ciftdogan ${ }^{1,2} \cdot$ Yildiz Ekemen Keles $^{2}$ (D) Benhur Sirvan Cetin ${ }^{3} \cdot$ Nazan Dalgic Karabulut $^{4} \cdot$ \\ Melike Emiroglu ${ }^{5}$.Zafer Bagci ${ }^{6}$. Ayse Buyukcam ${ }^{7}$. Emine Hafize Erdeniz ${ }^{8} \cdot$ Gul Arga $^{9} \cdot$ Edanur Yesil $^{10}$. \\ Ozlem Cakici ${ }^{11}$. Adem Karbuz ${ }^{12}$. Zumrut Sahbudak Bal ${ }^{13}$. Soner Sertan Kara ${ }^{14}$. Arife Ozer ${ }^{15}$. Ozge Metin Akcan ${ }^{16}$. \\ Sefika Elmas Bozdemir ${ }^{17}$. Ayse Berna Anil ${ }^{1}$. Hatice Uygun ${ }^{18} \cdot$ Omer Kilic $^{19}$. Selda Hancerli Torun ${ }^{20}$. Zuhal Umit ${ }^{21}$. \\ Murat Sutcu ${ }^{22}$. Berfin Ozgokce Ozmen ${ }^{23}$ • Hatice Karaoglu Asrak ${ }^{24}$. Gulsum Alkan ${ }^{5}$. Ahu Kara Aksay ${ }^{2}$. \\ Cuneyt Ugur ${ }^{6}$. Ahmet Ziya Birbilen ${ }^{7}$. Burcu Bursal Duramaz ${ }^{25}$. Esra Akyuz Ozkan ${ }^{8}$. Ozgur Burakay ${ }^{11}$. \\ Sema Yildirim Arslan $^{13}$. Eda Karadag Oncel ${ }^{2}$. Serkan Fazli Celik ${ }^{14}$. Ahmet Osman Kilic ${ }^{16} \cdot$ Seval Ozen $^{18}$. \\ Remzi Sarikaya ${ }^{15}$. Demet Demirkol ${ }^{20} \cdot$ Gazi Arslan $^{24}$ - Ozden Turel ${ }^{25} \cdot$ Ahmet Sert $^{5}$ - Ergul Sari ${ }^{26} \cdot$ Zerrin Orbak $^{27}$. \\ Irfan Oguz Sahin ${ }^{8}$. Celal Varan ${ }^{18} \cdot$ Hacer Akturk ${ }^{28} \cdot$ Sadiye Kubra Tuter Oz ${ }^{5} \cdot$ Fatih Durak $^{7} \cdot$ Mehmet Burhan Oflaz $^{16}$. \\ Manolya Kara ${ }^{22}$. Derya Karpuz ${ }^{23} \cdot$ Mey Talip Petmezci ${ }^{12} \cdot$ Nevin Hatipoglu $^{26} \cdot$ Selim Oncel ${ }^{11} \cdot$ Mehmet Turgut $^{18}$. \\ Ferhan Elmali ${ }^{1}$. Ayper Somer ${ }^{20} \cdot$ Necdet Kuyucu $^{23} \cdot$ Ener Cagri Dinleyici $^{19} \cdot$ Zafer Kurugöl $^{13} \cdot$ Ergin Ciftci $^{9}$. \\ Ates Kara ${ }^{29}$
}

Received: 15 September 2021 / Revised: 9 January 2022 / Accepted: 21 January 2022 / Published online: 7 February 2022 (c) The Author(s), under exclusive licence to Springer-Verlag GmbH Germany, part of Springer Nature 2022

\begin{abstract}
Multisystemic inflammatory syndrome (MIS-C) diagnosis remains difficult because the clinical features overlap with Kawasaki disease (KD). The study aims to highlight the clinical and laboratory features and outcomes of patients with MISC whose clinical manifestations overlap with or without $\mathrm{KD}$. This study is a retrospective analysis of a case series designed for patients aged 1 month to 18 years in 28 hospitals between November 1, 2020, and June 9, 2021. Patient demographics, complaints, laboratory results, echocardiographic results, system involvement, and outcomes were recorded. A total of 614 patients were enrolled; the median age was 7.4 years (interquartile range (IQR) 3.9-12 years). A total of 277 (45.1\%) patients with MIS-C had manifestations that overlapped with KD, including 92 (33.3\%) patients with complete $\mathrm{KD}$ and $185(66.7 \%)$ with incomplete KD. Lymphocyte and platelet counts were significantly lower in patients with MISC, overlapped with KD (lymphocyte count 1080 vs. 1280 cells $\times \mu \mathrm{L}, p=0.028$; platelet count 166 vs. 216 cells $\times 10^{3} / \mu \mathrm{L}, p<0.001$ ). The median serum procalcitonin levels were statistically higher in patients overlapped with $\mathrm{KD}(3.18 \mathrm{vs} .1 .68 \mu \mathrm{g} / \mathrm{L}, p=0.001)$. Coronary artery dilatation was statistically significant in patients with overlap with $\mathrm{KD}(13.4 \%$ vs. $6.8 \%, p=0.007)$, while myocarditis was significantly more common in patients without overlap with $\mathrm{KD}$ features $(2.6 \%$ vs $7.4 \%, p=0.009)$. The association between clinical and laboratory findings and overlap with KD was investigated. Age $>12$ years reduced the risk of overlap with KD by $66 \%(p<0.001$, 95\% CI $0.217-0.550)$, lethargy increased the risk of overlap with KD by 2.6 -fold $(p=0.011,95 \%$ CI 1.244-5.439), and each unit more albumin $(\mathrm{g} / \mathrm{dl})$ reduced the risk of overlap with $\mathrm{KD}$ by $60 \%(p<0.001,95 \% \mathrm{CI} 0.298-0.559)$.

Conclusion: Almost half of the patients with MISC had clinical features that overlapped with KD; in particular, incomplete $\mathrm{KD}$ was present. The median age was lower in patients with KD-like features. Lymphocyte and platelet counts were lower, and ferritin and procalcitonin levels were significantly higher in patients with overlap with KD.
\end{abstract}

\section{What is Known:}

- In some cases of MIS-C, the clinical symptoms overlap with Kawasaki disease.

- Compared to Kawasaki disease, lymphopenia was an independent predictor of MIS-C.

Communicated by Nicole Ritz

Extended author information available on the last page of the article 
What is New:

- Half of the patients had clinical features that overlapped with Kawasaki disease.

- In patients whose clinical features overlapped with KD, procalcitonin levels were almost 15 times higher than normal.

- Lethargy increased the risk of overlap with KD by 2.6-fold in MIS-C patients.

- Transient bradycardia was noted in approximately $10 \%$ of our patients after initiation of treatment.

Keywords MIS-C $\cdot$ Kawasaki disease $\cdot$ COVID-19 $\cdot$ Children

$\begin{array}{ll}\text { Abbreviations } \\ \text { MIS-C } & \text { Multisystemic inflammatory syndrome } \\ \text { KD } & \text { Kawasaki disease } \\ \text { SARS CoV-2 } & \text { Acute respiratory syndrome coronavirus 2 } \\ \text { BNP } & \text { Brain natriuretic peptide } \\ \text { NT-pro-BNP } & \text { N-terminal pro-B-type natriuretic peptide } \\ \text { ICU } & \text { Intensive care unit } \\ \text { ECMO } & \text { Extracorporeal membrane oxygenation } \\ \text { OR } & \text { Odds ratio } \\ \text { CI } & \text { Confidence interval } \\ \text { IQR } & \text { Interquartile range }\end{array}$

\section{Introduction}

The COVID-19 disease was first reported from Asia, with initial reports suggesting that children had only mild symptoms compared with adults [1]. However, in April 2020, the first case series from England was identified; then, many cases from Europe and the USA were defined as multisystemic inflammatory syndrome (MIS-C) associated with COVID-19, and it became clear that this disease does not present with mild symptoms in children, as previously thought [2-6]. Despite the growing awareness of MIS-C, diagnosis remains difficult because the clinical features overlap with many childhood diseases. In some cases, the clinical symptoms overlap with Kawasaki disease (KD), Kawasaki disease shock syndrome, or toxic shock syndrome, notably necessitating inotropic support and possibly a stay in the intensive care unit. Severe acute respiratory syndrome coronavirus 2 (SARS CoV-2) continues to spread worldwide, corresponding reports from Europe and the Americas and some reports from Asia in association with MISC are accumulating, and the clinical features are gradually being clarified [2-8].

It has been noted that MISC overlaps clinically with the features of KD, especially with incomplete Kawasaki disease [9]. The incidence of Kawasaki disease predominates in the first 5 years of life, in stark contrast to the epidemiology of MIS-C, which usually affects school-aged children $[6,10]$. The incidence of KD is highest in Asian countries, whereas more cases associated with MIS-C have been found in Western countries, particularly in African and Hispanic races [11-13]. Racial differences suggest that KD and MISC are caused by different pathophysiological and genetic susceptibilities. The clinical feature that distinguishes MIS-C from $\mathrm{KD}$ is myocardial involvement, resulting in significant myocardial dysfunction with marked increases in brain natriuretic peptide (BNP) and N-terminal pro-brain natriuretic peptide (Nt-ProBNP). In the most severely ill Kawasaki patients, coronary artery involvement and secondary cardiac dysfunction predominate; BNP and NT-ProBNP levels increase slightly $[14,15]$.

The aim of this study was to highlight the clinical and laboratory features and outcomes of patients with MISC whose clinical manifestations overlap with or without Kawasaki disease. It also aimed to highlight possible related laboratory and clinical features for MIS-C that are consistent with KD.

\section{Materials and methods}

\section{Study design}

This study is a retrospective analysis of a case series designed for patients aged 1 month to 18 years in 28 hospitals and 16 different cities in Turkey between November 1, 2020, and June 9,2021 . Patient demographics, underlying disease, medication history, complaints, laboratory results, system involvement, and outcomes were recorded in the medical records by completing the concept form. Laboratory and clinical parameters (lymphocyte count, neutrophil count, blood pressure, respiratory rate, and heart rate) were recorded as age-specific normal ranges. Echocardiographic findings, need for intensive care unit (ICU) due to inotropic support or fluid resuscitation, and need for invasive/non-invasive mechanical ventilation or extracorporeal membrane oxygenation (ECMO) were assessed. Treatment regimens and the time interval in which fever resolved after treatment were recorded. Patients who experienced complications and died were recorded. American Heart Association criteria were used to define incomplete and complete KD [10]. Patients were divided into two groups: patients with clinical findings overlapping with those of incomplete or complete $\mathrm{KD}$ and patients without such findings. The case definition of MIS-C was used as defined by the Centers for Disease Control and Prevention and World Health Organization [16, 17].

Inclusion criteria included evidence of SARS-CoV-2 infection by positive test results with RT-PCR or with serologic or 
antigen tests or a history of contact with a confirmed COVID19 patient. Thirteen patients were excluded because they did not meet the MIS-C case definition. Hospitals obtained ethics committee approval.

\section{Statistical analysis}

Descriptive statistics were presented for continuous variables as either mean or median depending on the distributional nature of the data. Categorical variables were presented as numbers and percentages. For comparison of categorical variables, the chi-square test or Fisher's exact test was used depending on the group, while for continuous variables in case of non-normal distribution, the Mann-Whitney $U$ test and Kruskal-Wallis test were used, and $p<0.05$ was considered significant. Univariate logistic regression analysis was used to assess the association of independent variables in patients with MIS-C as overlap with KD. Age groups, sex, underlying disease, complaints, clinical and laboratory findings, and need for ICU stay were included in the analysis to identify potential predictors of MIS-C patients with overlap with KD. Variables found to be statistically significant in univariate analysis $(p<0.250)$ were used in a multivariate logistic regression model with the stepwise backward Wald method to determine the independent factors of overlap risk in MIS-C patients with $\mathrm{KD}$. Odds ratio $(\mathrm{OR})$ and $95 \%$ confidence interval $(\mathrm{CI})$ were calculated. Statistical analyses were performed using SPSS software version 25 for Windows (IBM, Armonk, NY, USA).

\section{Results}

\section{Clinical characteristics of patients}

A total of 614 patients were enrolled in the study, and median age was 7.4 years (interquartile range (IQR) 3.9-12 years, range 30 days to 17.7 years). The demographic and clinical characteristics of the patients according to age groups are shown in Table 1. Most patients were boys $(57.7 \%, p<0.001)$. When the patients were compared according to age groups and sex, children $>12$ years were more likely to be boys than other age groups $(p=0.012$; Table 1). Most patients were previously healthy, and $11.8 \%(n=73)$ of the patients had an underlying disease; immunocompromised and autoimmune diseases were the most common $(n=19,3.1 \%)$, followed by neurometabolic $(n=17,2.8 \%)$ and respiratory diseases $(n=10,1.6 \%)$.

Median time from onset of symptoms to hospitalization was 4 days (IQR 2-5 days). All children presented with fever, which lasted a median of 5 days (IQR 4-6 days) and had a median temperature of $39{ }^{\circ} \mathrm{C}$ (IQR $38.5-39.3{ }^{\circ} \mathrm{C}$ ). Fatigue $(n=502,81.8 \%)$ and gastrointestinal symptoms were the most common complaints, with abdominal pain, vomiting, or diarrhea occurring in $77 \%(n=473)$ of patients. Fatigue and gastrointestinal complaints were found significantly less often in the $0-5$ age group than in the other age groups ( $p=0.009$ and $p=0.003$, respectively; Table 1). Clinical signs suggestive of KD- conjunctival injection $(n=305,49.7 \%)$, rash $(n=334,54.4 \%)$, and mucous membrane changes $(n=265,43.1 \%)$ were common. Conjunctival injections and mucous membrane changes, findings similar to KD, were found significantly more frequently in the 5-12 years age group than in the other groups (all for $p<0.001$; Table 1). Assessment of organ system involvement and age groups showed that in the $0-5$ years age group, $2-3$ systems were mainly involved, while in the other age groups, $4-5$ systems were more frequently involved ( $p<0.001$; Table 1$)$. Respiratory symptoms and the development of shock were statistically significantly more frequent in the $>12$ years group (all for $p<0.001$; Table 1).

\section{Demographic and laboratory findings in patients with MIS-C clinical manifestations with and without overlap with Kawasaki disease}

A total of 277 (45.1\%) patients with MIS-C had manifestations that appeared to overlap with KD, including 92 (33.3\%) patients with complete KD and 185 (66.7\%) with incomplete KD. The demographic and laboratory characteristics of the patients according to whether or not their clinical findings were consistent with KD are shown in Table 2. MIS-C patients with an overlap with KD were younger than patients without an overlap with KD (7 years vs. 8 years, respectively, $p=0.021$ ). When comparing the two groups in terms of SARS-CoV-2 test results, children with MIS-C who had an overlap with KD had numerically higher RT-PCR test positivity ( $13.3 \%$ vs. $5.7 \%, p=0.001)$, whereas in patients without an overlap with $\mathrm{KD}$, only serological test positivity was significant $(87.6 \%$ vs. $92.7 \%$, $p=0.041$ ).

Laboratory results showed that, in particular, lymphocyte and platelet counts were significantly lower in patients with MISC, whose features overlapped with KD (lymphocyte count 1080 vs. 1280 cells $\times \mu \mathrm{L}, p=0.028$; platelet count 166 vs. 216 cells $\left.\times 10^{3} / \mu \mathrm{L}, p<0.001\right)$. Significantly elevated inflammatory markers were found, such as elevated CRP $(\mathrm{CRP} \geq 100 \mathrm{mg} / \mathrm{L}$ in $67.9 \%$ (417/614)), elevated procalcitonin (procalcitonin $\geq 0.2 \mu \mathrm{g} / \mathrm{L}$ in $89.6 \%$ (489/546)), elevated erythrocyte sedimentation rate (ESR $\geq 40 \mathrm{~mm} / \mathrm{h}$ in $53.1 \%$ (260/490)), and elevated ferritin (ferritin $\geq 400 \mu \mathrm{g} / \mathrm{L}$ in $39.2 \%(233 / 594))$. When comparing acute-phase reactants in children whose clinical features overlapped with or without Kawasaki disease, median serum procalcitonin and ferritin levels were statistically higher in patients that overlapped 
Table 1 Demographic and clinical characteristics according to specific age range of the patients with MIS-C

\begin{tabular}{|c|c|c|c|c|c|c|c|c|}
\hline \multirow[t]{2}{*}{ Characteristic } & \multirow{2}{*}{$\begin{array}{l}\text { All Patients } \\
n=614\end{array}$} & \multirow{2}{*}{$\begin{array}{l}0-5 \text { years } \\
n=205\end{array}$} & \multirow{2}{*}{$\begin{array}{l}5-12 \text { years } \\
n=255\end{array}$} & \multirow{2}{*}{$\begin{array}{l}>12 \text { years } \\
n=154\end{array}$} & \multirow[t]{2}{*}{$p$ value } & \multicolumn{3}{|l|}{$p$ value } \\
\hline & & & & & & $\begin{array}{l}0-5 \mathrm{yr} \\
\text { vs } \\
5-12 \mathrm{yr}\end{array}$ & $\begin{array}{l}0-5 \mathrm{yr} \\
\mathrm{vs} \\
>12 \mathrm{yr}\end{array}$ & $\begin{array}{l}5-12 \mathrm{yr} \\
\mathrm{vs} \\
>12 \mathrm{yr}\end{array}$ \\
\hline Age. median (IQR) $)^{\mathrm{a}}$ year & $7.4(3.9-12)$ & $2.9(1.7-4)$ & $8(6.4-10)$ & $14.3(13-15.9)$ & - & - & - & - \\
\hline Sex, no (\%) & & & & & 0.012 & 0.406 & 0.003 & 0.022 \\
\hline Boy & $354(57.7)$ & $107(52.2)$ & $143(56.1)$ & $104(67.5)$ & - & - & - & - \\
\hline Girl & $260(42.3)$ & $98(47.8)$ & $112(43.9)$ & $50(32.5)$ & & - & - & - \\
\hline Race/ethnicity (\%) & & & & & 0.450 & - & - & - \\
\hline Turkish & $590(96.1)$ & $195(95.1)$ & $248(97.3)$ & $147(95.5)$ & - & - & - & - \\
\hline Others & $24(3.9)$ & $10(4.9)$ & $7(2.7)$ & $7(4.5)$ & - & - & - & - \\
\hline $\begin{array}{l}\text { Overweight/obese }{ }^{\mathrm{b}} \text { patient no-total } \\
\text { no }(\%)\end{array}$ & $124 / 494(25.1)$ & $36 / 157(22.9)$ & $58 / 209(27.8)$ & $30 / 128(23.4)$ & 0.506 & - & - & \\
\hline $\begin{array}{l}\text { Underlying medical condition } \\
\text { (any) (\%) }\end{array}$ & $73(11.8)$ & $18(8.8)$ & $33(12.9)$ & $22(14.3)$ & 0.221 & - & - & - \\
\hline $\begin{array}{l}\text { Immunocompromising or autoim- } \\
\text { mune }\end{array}$ & $19(3.1)$ & $4(2)$ & $6(2.4)$ & $9(5.8)$ & 0.089 & - & - & - \\
\hline Neurometabolic disease & $17(2.8)$ & $7(3.4)$ & $4(1.6)$ & $6(3.9)$ & 0.327 & - & - & - \\
\hline Respiratory & $10(1.6)$ & $1(0.5)$ & $7(2.7)$ & $2(1.3)$ & $0.161^{\mathrm{c}}$ & - & - & - \\
\hline Cardiac & $7(1.1)$ & $3(1.5)$ & $4(1.6)$ & 0 & $0.343^{\mathrm{c}}$ & - & - & - \\
\hline $\begin{array}{l}\text { Number of organ systems involve- } \\
\text { ments n }(\%)\end{array}$ & & & & & $<0.001$ & $<0.001$ & $<0.001$ & 0.001 \\
\hline $2-3$ & $256(41.7)$ & $112(54.6)$ & $90(35.3)$ & $54(35.1)$ & - & - & - & - \\
\hline $4-5$ & $307(50)$ & $85(41.5)$ & $149(58.4)$ & $73(47.4)$ & - & - & - & - \\
\hline $6 \geq$ & $51(8.3)$ & $8(3.9)$ & $16(6.3)$ & $27(17.5)$ & - & - & - & - \\
\hline \multicolumn{9}{|l|}{ Clinical features at presentation } \\
\hline Fever, $n(\%)$ & $614(100)$ & $205(100)$ & $255(100)$ & $154(100)$ & $0.266^{\mathrm{c}}$ & - & - & - \\
\hline Degree of fever $\left({ }^{\circ} \mathrm{C}\right)$ median $(\mathrm{IQR})$ & $39(38.5-39.3)$ & $39(38.5-39.3)$ & $39(38.5-39.5)$ & $38.9(38.4-39)$ & 0.005 & 1.000 & 0.040 & $\mathbf{0 . 0 3 8}$ \\
\hline $\begin{array}{l}\text { Duration of fever (day) median } \\
\text { (IQR) }\end{array}$ & $5(4-6)$ & $5(3-6)$ & $5(4-7)$ & $5(3-6)$ & 0.179 & - & - & - \\
\hline Fatigue, $n(\%)$ & $502(81.8)$ & $154(75.1)$ & $219(85.9)$ & $129(83.8)$ & 0.009 & 0.003 & 0.047 & 0.561 \\
\hline $\begin{array}{l}\text { Any gastrointestinal symptoms, } n \\
(\%)\end{array}$ & $473(77)$ & $141(68.8)$ & $207(81.2)$ & $125(81.2)$ & 0.003 & 0.002 & 0.008 & 0.998 \\
\hline $\begin{array}{l}\text { Abdominal pain patient no-total no } \\
(\%)\end{array}$ & $322 / 501(64.3)$ & $50 / 94(53.2)$ & $168 / 253(66.4)$ & $104(67.5)$ & 0.044 & 0.024 & 0.024 & 0.814 \\
\hline Vomiting, $n(\%)$ & $286(46.6)$ & $82(40)$ & $130(51)$ & $74(48.1)$ & 0.058 & - & - & \\
\hline Nausea patient no-total no (\%) & $259(42.2)$ & $70(34.4)$ & $115(45.1)$ & $74(48.1)$ & 0.014 & 0.017 & 0.008 & 0.562 \\
\hline Diarrhea, $n(\%)$ & $259(42.2)$ & $87(42.4)$ & $110(43.1)$ & $62(40.3)$ & 0.846 & - & - & - \\
\hline Conjunctival injection $\mathrm{n}(\%)$ & $305(49.7)$ & $90(43.9)$ & $154(60.4)$ & $61(39.6)$ & $<0.001$ & $<0.001$ & 0.415 & $<0.001$ \\
\hline Rash, $n(\%)$ & $334(54.4)$ & $114(55.6)$ & $148(58)$ & $72(46.8)$ & 0.078 & - & - & - \\
\hline Maculopapular & $217(35.3)$ & $71(34.6)$ & $99(38.8)$ & $47(30.5)$ & - & - & - & - \\
\hline Macule & $87(14.2)$ & $30(14.6)$ & $41(16.1)$ & $16(10.4)$ & - & - & - & - \\
\hline Petechiae/ecchymosis & $8(1.3)$ & $2(1)$ & $3(1.2)$ & $3(1.9)$ & - & - & - & - \\
\hline Others & $22(3.6)$ & $11(5.4)$ & $5(2)$ & $6(3.9)$ & - & - & - & - \\
\hline Mucous membrane changes, $n(\%)$ & $265(43.1)$ & $90(43.9)$ & $130(51)$ & $45(29.2)$ & $<0.001$ & 0.131 & 0.004 & $<0.001$ \\
\hline Any Respiratory symptoms, $n(\%)$ & $213(34.6)$ & $54(26.6)$ & $80(31.4)$ & $79(51.3)$ & $<0.001$ & 0.238 & $<0.001$ & $<0.001$ \\
\hline Muscle ache patient no-total no (\%) & $179 / 531(33.7)$ & $18 / 124(14.5)$ & $80 / 253(31.6)$ & $81 / 154(52.6)$ & $<0.001$ & $<0.001$ & $<0.001$ & $<0.001$ \\
\hline Headache patient no-total no (\%) & $119 / 477(24.9)$ & $13 / 70(18.6)$ & $60 / 253(23.7)$ & $46 / 154(29.9)$ & 0.156 & - & - & - \\
\hline $\begin{array}{l}\text { Peripheral cutaneous inflammation } \\
\text { signs, } n(\%)\end{array}$ & $116(18.9)$ & $49(23.9)$ & $52(20.4)$ & $15(9.7)$ & 0.002 & 0.366 & $<0.001$ & 0.005 \\
\hline Dry cough n $(\%)$ & $96(15.6)$ & $23(11.2)$ & $34(13.3)$ & $39(25.3)$ & 0.001 & 0.494 & $<0.001$ & 0.002 \\
\hline Shock, $n(\%)$ & $74(12.1)$ & $10(4.9)$ & $30(11.8)$ & $34(22.1)$ & $<0.001$ & 0.009 & $<0.001$ & 0.005 \\
\hline
\end{tabular}


Table 1 (continued)

\begin{tabular}{|c|c|c|c|c|c|c|c|c|}
\hline \multirow[t]{2}{*}{ Characteristic } & \multirow{2}{*}{$\begin{array}{l}\text { All Patients } \\
n=614\end{array}$} & \multirow{2}{*}{$\begin{array}{l}0-5 \text { years } \\
n=205\end{array}$} & \multirow{2}{*}{$\begin{array}{l}5-12 \text { years } \\
n=255\end{array}$} & \multirow{2}{*}{$\begin{array}{l}>12 \text { years } \\
n=154\end{array}$} & \multirow[t]{2}{*}{$p$ value } & \multicolumn{3}{|l|}{$p$ value } \\
\hline & & & & & & $\begin{array}{l}0-5 \mathrm{yr} \\
\text { vs } \\
5-12 \mathrm{yr}\end{array}$ & $\begin{array}{l}0-5 \mathrm{yr} \\
\mathrm{vs} \\
>12 \mathrm{yr}\end{array}$ & $\begin{array}{l}5-12 \mathrm{yr} \\
\text { vs } \\
>12 \mathrm{yr}\end{array}$ \\
\hline Sore throat patient no-total no $(\%)$ & $71 / 492(11.6)$ & $9 / 85(10.6)$ & $35 / 253(13.8)$ & $27 / 154(17.5)$ & 0.318 & - & - & - \\
\hline Arthralgia patient no-total no (\%) & $48 / 493(9.7)$ & $6 / 86(7)$ & $22 / 253(8.7)$ & $20 / 154(13)$ & 0.241 & - & - & \\
\hline Lethargy, $n(\%)$ & $53(8.6)$ & $20(9.8)$ & $23(9)$ & $10(6.5)$ & 0.530 & - & - & - \\
\hline Lymphadenopathy, $n(\%)$ & $49(8)$ & $20(9.8)$ & $23(9)$ & $6(3.9)$ & 0.093 & - & - & - \\
\hline Desquamation, $n(\%)$ & $34(5.5)$ & $14(6.8)$ & $12(4.7)$ & $8(5.2)$ & 0.642 & - & - & - \\
\hline Neck stiffness, $n(\%)$ & $32(5.2)$ & $3(1.5)$ & $19(7.5)$ & $10(6.5)$ & 0.012 & 0.012 & 0.003 & 0.012 \\
\hline Taste loss. patient no-total no (\%) & $16 / 476(3.4)$ & - & $5(2)$ & $11(7.1)$ & $0.007^{\mathrm{c}}$ & $0.359^{\mathrm{c}}$ & $0.039^{c}$ & 0.010 \\
\hline Runny nose, $n(\%)$ & $21(3.4)$ & $10(4.9)$ & $5(2)$ & $6(3.9)$ & 0.216 & - & - & - \\
\hline Arthritis, $n(\%)$ & $18(2.9)$ & $6(2.9)$ & $8(3.1)$ & $4(2.6)$ & 0.956 & - & - & - \\
\hline Convulsion, $n(\%)$ & $17(2.8)$ & $8(3.9)$ & $3(1.2)$ & $6(3.9)$ & 0.128 & - & - & - \\
\hline Paralysis, $n(\%)$ & $13(2.1)$ & $3(1.5)$ & $4(1.6)$ & $6(3.9)$ & $0.214^{\mathrm{c}}$ & - & - & - \\
\hline Ileus, $n(\%)$ & $8(1.3)$ & 0 & $4(1.6)$ & $4(2.6)$ & 0.062 & - & - & - \\
\hline $\begin{array}{l}\text { Loss of smell. patient no-total no } \\
(\%)\end{array}$ & $7 / 476(1.5)$ & - & $2(0.8)$ & $5(3.2)$ & $0.131^{\mathrm{c}}$ & - & - & - \\
\hline Odynophagia, $n(\%)$ & $4 / 483(0.8)$ & - & $2(0.8)$ & $2(1.3)$ & $0.651^{\mathrm{c}}$ & - & - & - \\
\hline
\end{tabular}

${ }^{\mathrm{a}} \mathrm{IQR}$, interquartile range

${ }^{\mathrm{b}}$ Overweight is defined as a body mass index (BMI) at or above the 85th percentile and below 95th percentile for children with MIS-C. Obesity was described as a BMI at or above the 95th percentile of the same age and gender children over 2 years old. Under 2 years of age, World Health organization Child Growth Standards were used to define overweight and obesity

${ }^{c}$ Fisher's exact probability test was used for cross-classification tables

with KD (procalcitonin 3.18 vs. $1.68 \mu \mathrm{g} / \mathrm{L}, p=0.001$; ferritin 396 vs. $258.5 \mu \mathrm{g} / \mathrm{L}, p<0.001)$.

\section{System involvement and outcomes}

Clinical outcomes, treatments, and echocardiographic findings of the patients are listed in Table 3 . The most commonly involved systems were the hematologic and cardiovascular systems. When the correlation between the number of organ systems affected and age was evaluated, there was a significant weak correlation between the number of organ systems affected and the age of the patients $(\mathrm{rho}=0.254, p<0.001)$.

Intensive care unit stay was required in $31.3 \%(n=192)$, and shock occurred in $12.1 \%$ of patients. Interestingly, patients without an overlap with a KD required a higher number of ICU stays $(23.8 \%$ vs. $37.4 \%, p<0.001)$ and development of shock (9.7\% vs. 13.9\%) (Table 3). Median length of hospital stay was 9 days (IQR 6-12 days) and the median length of ICU stay was 4 days (IQR 2-7 days).

Echocardiography results were evaluated for both groups. Coronary artery dilatation was found in $9.9 \%$ $(n=57 / 578)$ of patients, in $13.4 \%(n=36 / 268)$ of the group whose clinical findings overlapped with $\mathrm{KD}$, and in $6.8 \%(21 / 310)$ of the other group $(p=0.007)$. Coronary artery aneurysm was found in five patients, four of whom were in the group with KD-like features. The frequency of coronary artery aneurysm was not statistically significant between the two groups $(p=0.188)$. Valvulitis was statistically significant in patients with overlap with $\mathrm{KD}$, while myocarditis was significantly more common in patients without overlap with KD features $(p=0.002, p=0.009$, respectively) (Table 3).

Majority of the patients received intravenous immunoglobulin $(n=571,93 \%)$. This treatment was followed by corticosteroids $(n=514,83.8 \%)$, acetylsalicylic acid $(n=409,66.6 \%)$, and anticoagulants $(n=368,59.9 \%)$. Median time from initiation of IVIG therapy to relief of fever was $24 \mathrm{~h}(n=287$, IQR 8-24 h). Similarly, the time from initiation of intravenous corticosteroids to relief of fever was $24 \mathrm{~h}(n=412$, IQR $8-24 \mathrm{~h})$. IVIG and ASA treatments were statistically significantly more frequently administered in patients with clinical features overlapping with $\mathrm{KD}$, whereas inotropes were frequently administered in patients not overlapping with $\mathrm{KD}$ (Table 3 ).

Interestingly, sinus bradycardia was noted in 64 (10.4\%) patients. Median time to onset of bradycardia was 3 days (IQR 2-4 days) from the start of treatment, while median duration of bradycardia was 2 days (IQR 2-3 days). IVIG treatment was given in $96.9 \%$ of patients who developed bradycardia, and intravenous corticosteroid was administered in $90.6 \%$ of these patients. Two of these patients were monitored in the ICU, and a Holter electrocardiogram was performed. Bradycardia resolved without treatment. 
Table 2 Demographic and laboratory characteristics of the patients by overlapping with Kawasaki disease or not

\begin{tabular}{|c|c|c|c|c|}
\hline Characteristic & $\begin{array}{l}\text { All patients } \\
n=614\end{array}$ & $\begin{array}{l}\text { Overlapping with Kawa- } \\
\text { saki disease } \\
n=277\end{array}$ & $\begin{array}{l}\text { Others } \\
n=337\end{array}$ & $p$ value \\
\hline Age. median (IQR) $)^{\mathrm{a}}$ year & $7.4(3.9-12)$ & $7(3.8-10.5)$ & $8(3.9-13)$ & 0.021 \\
\hline \multicolumn{5}{|l|}{ Sex, no $(\%)$} \\
\hline Boy & $354(57.7)$ & $164(59.2)$ & $190(56.4)$ & \multirow[t]{2}{*}{0.481} \\
\hline Girl & $260(42.3)$ & $113(40.8)$ & $147(43.6)$ & \\
\hline \multicolumn{5}{|l|}{ Laboratory characteristics } \\
\hline \multicolumn{5}{|l|}{ SARS CoV-2 testing } \\
\hline Serology positive/PCR negative ${ }^{\mathrm{b}}$ (among tested) & $498(90.3)$ & $218(87.6)$ & $280(92.7)$ & 0.041 \\
\hline PCR positive/serology negative or not done & $34(5.5)$ & $22(7.9)$ & $12(3.6)$ & 0.018 \\
\hline PCR positive/serology positive ${ }^{\mathrm{b}}$ (among tested) & $22(3.9)$ & $15(5.4)$ & $7(2.1)$ & 0.027 \\
\hline $\begin{array}{l}\text { Epidemiologic link only, PCR negative and serology negative or } \\
\text { serology not done }\end{array}$ & $60(9.8)$ & $22(7.9)$ & $38(11.3)$ & 0.166 \\
\hline \multicolumn{5}{|l|}{ Hematology } \\
\hline Total white blood cell count cells $\times 10^{3} / \mu \mathrm{L}$, median (IQR) & $9500(6380-13,900)$ & $9000(6150-12,905)$ & $9920(6520-14,960)$ & 0.012 \\
\hline Neutrophil count cells $\times 10^{3} / \mu \mathrm{L}$ median $(\mathrm{IQR})$ & $7220(4397-10,785)$ & $7240(4370-10,030)$ & $7200(4427-11,595)$ & 0.109 \\
\hline Lymphocyte count cells $\times 10^{3} / \mu \mathrm{L}$, median (IQR) & $1180(700-2010)$ & $1080(640-2000)$ & $1280(780-2035)$ & 0.028 \\
\hline Platelet count cells $\times 10^{3} / \mu \mathrm{L}$, median (IQR) & $190(131-285)$ & $166(119-242)$ & $216(152-301)$ & $<0.001$ \\
\hline Hemoglobin $\mathrm{g} / \mathrm{dL}$, median (IQR) & $11.5(10.5-12.5)$ & $11.4(10.3-12.3)$ & $11.5(10.6-12.6)$ & 0.186 \\
\hline Eosinophil count cells $/ \mu \mathrm{L}$, median (IQR) & $70(10-170)$ & $80(10-200)$ & $50(10-150)$ & 0.047 \\
\hline \multicolumn{5}{|l|}{ Inflammatory markers } \\
\hline $\mathrm{CRP}, \mathrm{mg} / \mathrm{L}$, median (IQR) & $140(83-207)$ & $137.9(80-207)$ & $142(85.5-205)$ & 0.625 \\
\hline $\mathrm{ESR}, \mathrm{mm} / \mathrm{h}$, median (IQR) $(n)$ & $\begin{array}{l}42(22-69.2) \\
{[490]}\end{array}$ & $\begin{array}{l}43(25-70) \\
{[240]}\end{array}$ & $\begin{array}{l}40.5(21-69) \\
{[250]}\end{array}$ & 0.370 \\
\hline Procalcitonin, $\mu \mathrm{g} / \mathrm{L}$, median (IQR) $(n)$ & $\begin{array}{l}2(0.54-9) \\
{[546]}\end{array}$ & $\begin{array}{l}3.18(0.71-14) \\
{[252]}\end{array}$ & $\begin{array}{l}1.68(0.44-6.07) \\
{[294]}\end{array}$ & 0.001 \\
\hline Ferritin, $\mu \mathrm{g} / \mathrm{L}$, median (IQR) $(n)$ & $\begin{array}{l}302(147-576) \\
{[594]}\end{array}$ & $\begin{array}{l}396(193.5-725) \\
{[266]}\end{array}$ & $\begin{array}{l}258.5(116.2-476) \\
{[328]}\end{array}$ & $<0.001$ \\
\hline \multicolumn{5}{|l|}{ Biochemistry } \\
\hline LDH, U/L, median (IQR) & $299(242-355)$ & $308(255.2-360.7)$ & $290(235-351)$ & 0.026 \\
\hline AST, median, $\mu / \mathrm{L}(\mathrm{IQR})$ & $30(23-47)$ & $31(23.3-50.5)$ & $29(22-44)$ & 0.093 \\
\hline ALT, median, $\mu / L(I Q R)$ & $22(14-41)$ & $25(16-43.2)$ & $20(13-39.5)$ & 0.001 \\
\hline Albumin, g/dL, median (IQR) & $3.43(3-3.9)$ & $3.2(2.8-3.7)$ & $3.6(3.2-4)$ & $<0.001$ \\
\hline Sodium, mmol/L, median (IQR) $(n)$ & $\begin{array}{l}134(132-137) \\
{[563]}\end{array}$ & $\begin{array}{l}134(131-137) \\
{[267]}\end{array}$ & $\begin{array}{l}135(132-137) \\
{[296]}\end{array}$ & 0.025 \\
\hline Urea, mg/dL, median (IQR) $(n)$ & $\begin{array}{l}19(13-27) \\
{[613]}\end{array}$ & $\begin{array}{l}20(14-28.9) \\
{[277]}\end{array}$ & $\begin{array}{l}18(13-26) \\
{[336]}\end{array}$ & 0.078 \\
\hline Creatinine, $\mathrm{mg} / \mathrm{dL}$, median (IQR) $(n)$ & $\begin{array}{l}0.48(0.36-0.64) \\
{[603]}\end{array}$ & $\begin{array}{l}0.45(0.36-0.58) \\
{[271]}\end{array}$ & $\begin{array}{l}0.5(0.36-0.69) \\
{[332]}\end{array}$ & 0.012 \\
\hline Triglyceride, mg/dL, median (IQR) (n) & $\begin{array}{l}156(105-231) \\
{[278]}\end{array}$ & $\begin{array}{l}184(120-248) \\
{[131]}\end{array}$ & $\begin{array}{l}131(98-195) \\
{[147]}\end{array}$ & $<0.001$ \\
\hline \multicolumn{5}{|l|}{ Cardiac markers } \\
\hline Troponin, ng/L, median (IQR) $(n)$ & $\begin{array}{l}10(4-33) \\
{[597]}\end{array}$ & $\begin{array}{l}11(6.15-31.9) \\
{[266]}\end{array}$ & $\begin{array}{l}10(3-34) \\
{[331]}\end{array}$ & 0.007 \\
\hline NT-pro-BNP, pg/mL, median $(n)$ & $\begin{array}{l}1420(355-5193) \\
{[353]}\end{array}$ & $\begin{array}{l}2435(693-6975) \\
{[141]}\end{array}$ & $\begin{array}{l}966(276-4117) \\
{[212]}\end{array}$ & $<0.001$ \\
\hline BNP, ng/L, median (IQR) $(n)$ & $\begin{array}{l}598(89-5210) \\
{[91]}\end{array}$ & $\begin{array}{l}1606(184-6731) \\
{[41]}\end{array}$ & $\begin{array}{l}298(64-3619) \\
{[50]}\end{array}$ & 0.098 \\
\hline \multicolumn{5}{|l|}{ Coagulation } \\
\hline Fibrinogen, $\mathrm{mg} / \mathrm{dL}$, median (IQR) $(n)$ & $\begin{array}{l}501(399-622.2) \\
{[534]}\end{array}$ & $\begin{array}{l}480(383-589) \\
{[230]}\end{array}$ & $\begin{array}{l}511.1(418.9-637.6) \\
{[304]}\end{array}$ & 0.022 \\
\hline D-dimer, $\mu \mathrm{g} / \mathrm{L}$, median (IQR) $(n)$ & $\begin{array}{l}2320(1122-4241) \\
{[605]}\end{array}$ & $\begin{array}{l}2920(1414-4814) \\
{[273]}\end{array}$ & $\begin{array}{l}1940(1021-3880) \\
{[332]}\end{array}$ & $<0.001$ \\
\hline APTZ, median, sc (IQR) $(n)$ & $\begin{array}{l}28.3(25.4-32.7) \\
{[553]}\end{array}$ & $\begin{array}{l}29.3(26.2-33.8) \\
{[231]}\end{array}$ & $\begin{array}{l}27.7(24.6-31.8) \\
{[322]}\end{array}$ & $<0.001$ \\
\hline
\end{tabular}


Table 2 (continued)

\begin{tabular}{llll}
\hline Characteristic & $\begin{array}{l}\text { All patients } \\
n=614\end{array}$ & $\begin{array}{l}\text { Overlapping with Kawa- } \\
\text { saki disease } \\
n=277\end{array}$ \\
\hline PZ, median, sc (IQR) $(n)$ & $\begin{array}{l}13.9(12.5-15.6) \\
{[520]}\end{array}$ & $\begin{array}{l}13.7(12.4-15.5) \\
{[235]}\end{array}$ & $\begin{array}{l}0.245(12.8-15.8) \\
{[285]}\end{array}$ \\
\hline
\end{tabular}

${ }^{\mathrm{a}} \mathrm{IQR}$, interquartile range

${ }^{\mathrm{b}}$ Percentages calculated among 551 patients whose had serologic test results for SARS CoV-2

Eleven patients (1.8\%) died. Five of these patients had concomitant diseases; two suffered from acute lymphoblastic leukemia, one from aplastic anemia, one from ataxia telangiectasia, and one from congenital cytomegalovirus disease. Mean age of the deceased patients was 11.5 years $( \pm 5$, range 2.3-17 years). While six deceased patients received immunomodulatory treatment, two patients were treated with an extracorporeal membrane oxygenator.

Univariate logistic regression analysis was used to determine the independent risk predictors for patients with MIS-C whose clinical characteristics overlapped with KD (Table 4). Age groups, nausea, fatigue, lethargy, lymphocyte count, neutrophil count, hemoglobin level, platelet count, alanine aminotransferase, ferritin level, D-dimer level, and albumin level were statistically significant. The multivariate model was adjusted for these predictors, and the final model is shown in Table 5. Compared to age 5-12 years, the risk of overlap with KD decreased by $65.5 \%$ at age $>12$ years $(p<0.001,95 \%$ CI $0.217-0.550)$. Clinical findings and laboratory values were examined for risk of overlap with KD. Lethargy increased the risk of overlap with Kawasaki disease by 2.6 -fold ( $p=0.01195 \%$ CI 1.244-5.439) and each unit more of albumin $(\mathrm{g} / \mathrm{dL})$ reduced the risk of overlap with Kawasaki disease by $60 \%(p<0.00195 \%$ CI $0.298-0.559)$.

\section{Discussion}

In the present series, we compared the clinical features, laboratory findings, treatment methods, and outcomes of patients with MIS-C from multiple centers with or without overlap with KD. Some aspects of MIS-C resemble KD, such as prolonged fever, mucocutaneous inflammatory signs, rash, lymphadenopathy, and high inflammatory markers. We found that nearly half of the patients had clinical features that overlapped with KD.

In a large case series of MIS-C, $60 \%$ of the patients were male; median age of the patients with MIS-C was 9 years; half of the children with MIS-C were aged 5-13 years [18]. Consistent with the literature, we found that $57.7 \%$ of patients with MIS-C were male $[9,18]$. Also, the median age was 7.5 years. As expected, these results show that patients with MIS-C are more common in school-aged children. One quarter of our patients were obese, a finding consistent with most MIS-C series [5, 6, 9].
Patients' complaints were compared by age group; fatigue and gastrointestinal complaints were significantly more common in patients aged $\geq 5$ years. We suspected that this might be due to the difficulty in expressing these complaints at a younger age. We also found that respiratory symptoms and the development of shock were significantly more common at $>12$ years of age. We observed that conjunctival injection, rash, and mucous membrane changes reminiscent of KD were more common, especially in children under 12 years of age.

In the study by Feldstein et al., clinical manifestations have overlapped with $\mathrm{KD}$ in $40 \%$ of patients [6]. In our case series, there was an overlap with $\mathrm{KD}$ in almost half $(n=277,45.1 \%)$ of the patients, and two-thirds of these patients had an overlap with incomplete KD. In contrast to these findings, some studies have found that clinical manifestations that overlap with KD are significantly less common $[4,9]$. In a case series from the USA, 4.9\% (28/570) of the patients had clinical features that overlapped with complete $\mathrm{KD}$ [9]. In another study from England, 22\% (13/58) of the patients had Kawasaki-like clinical features [4]. Although it is difficult to explain the reason for the high rate of clinical features of MIS-C overlapping with $\mathrm{KD}$, it is thought to be due to racial genetic differences. Overlapping clinical features and the lack of a definitive diagnostic test may complicate the distinction between $\mathrm{KD}$ and MIS-C. In addition, due to the gradual increase in seroconversion rates to SARS-CoV-2 in the general population, diagnostic confusion can be expected when suspicion of $\mathrm{KD}$ is based on the positivity of antibodies, antigens, or RT-PCR to SARS-CoV-2.

Godfred-Cato et al. have reported a case series of MIS-C from the USA, with most $(n=490,86 \%)$ patients having four or more organ systems affected [9]. This is consistent with our series in which patients $(n=358,58 \%)$ had four or more organ systems affected. In this MIS-C cohort, coronary artery dilatation or aneurysms were found in $18.6 \%$ of cases [9]. In a study by Feldestien et al., coronary aneurysms were found in $8.8 \%$ of patients [6]. In the study by Jain et al., coronary artery dilatation was found in $23 \%(n=6 / 23)$ of patients with MIS-C [8]. In one of the publications with higher coronary artery aneurysm rates, coronary artery aneurysms were found 33\% (4/12) of the patients [19]. In our study, coronary artery dilatations and aneurysms were found in $10.8 \%$ of patients. Coronary artery dilatations were found significantly more frequently in the group whose clinical findings overlapped with $\mathrm{KD}$ ( $13 \%$ vs. $6.8 \%$, respectively, $p=0.007)$. Four of the five 
Table 3 Clinical outcomes, echocardiography results, and management of the patients with MIS-C by overlapping with Kawasaki disease or not

$\begin{array}{llll}\begin{array}{l}\text { All patients } \\ n=614\end{array} & \begin{array}{l}\text { Overlapping with Kawasaki } \\ \text { disease }^{\mathrm{a}} n=277\end{array} & \begin{array}{l}\text { Others } \\ n=337\end{array} & p \text { value }\end{array}$

\begin{tabular}{|c|c|c|c|c|}
\hline \multicolumn{5}{|l|}{ Characteristic } \\
\hline $\mathrm{ICU}_{\text {admission }}^{\mathrm{b}}, \underline{n}(\%)$ & $192(31.3)$ & $66(23.8)$ & $126(37.4)$ & $<0.001$ \\
\hline Duration of ICU days, median $(\mathrm{IQR})^{\mathrm{c}}$ & $4(2-7)$ & $4(2-7)$ & $4(2-7)$ & 0.859 \\
\hline Duration of hospitalization, median (IQR) & $9(6-12)$ & $9(6-12)$ & $8(6-11)$ & 0.157 \\
\hline Respiratory failure $^{\mathrm{d}}, n(\%)$ & $76(12.4)$ & $35(12.6)$ & $41(12.2)$ & 0.861 \\
\hline Hepatic failure $^{\mathrm{e}}, n(\%)$ & $22(3.6)$ & $17(6.1)$ & $5(1.5)$ & 0.002 \\
\hline Acute kidney injury ${ }^{\mathrm{f}}, n(\%)$ & $33(5.4)$ & $13(4.7)$ & $20(5.9)$ & 0.497 \\
\hline Hematologic failure ${ }^{\mathrm{g}}, n(\%)$ & $586(95.4)$ & $265(95.7)$ & $321(95.3)$ & 0.806 \\
\hline Macrophage activation syndrome ${ }^{\mathrm{h}}, n(\%)$ & $25(4.1)$ & $20(7.2)$ & $5(1.5)$ & $<0.001$ \\
\hline Gastrointestinal involvement ${ }^{\mathrm{i}}, n(\%)$ & $512(83.4)$ & $225(81.2)$ & $287(85.2)$ & 0.192 \\
\hline Neurological involvement ${ }^{\mathrm{j}}, n(\%)$ & $170(27.7)$ & $98(35.4)$ & $72(21.4)$ & $<0.001$ \\
\hline Cardiovascular involvement ${ }^{\mathrm{k}}, \boldsymbol{n}(\%)$ & $464(75.6)$ & $210(75.8)$ & $254(75.4)$ & 0.899 \\
\hline Shock (inotropes or vasopressors) ${ }^{1}, n(\%)$ & $74(12.1)$ & $27(9.7)$ & $47(13.9)$ & 0.112 \\
\hline Elevated troponin, $n(\%)$ & 98/597 (16.4) & $38 / 266(14.3)$ & $60 / 331(18.1)$ & 0.208 \\
\hline Elevated BNP, $n(\%)$ & 49/91 (53.8) & $26 / 41(63.4)$ & $23 / 50(46)$ & 0.097 \\
\hline Elevated NT_pro BNP, $n(\%)$ & $256 / 353(72.5)$ & $117 / 141(83)$ & $139 / 212(65.6)$ & $<0.001$ \\
\hline Abnormal Electrocardiogram, $n(\%)$ & $97 / 569(17)$ & $57 / 273(20.9)$ & $40 / 296(13.5)$ & 0.020 \\
\hline \multicolumn{5}{|l|}{ Echocardiography results } \\
\hline Ejection fraction, median (IQR) & $65(60-70)$ & $65(60-70)$ & $65(60-70)$ & 0.988 \\
\hline Congestive heart failure, patient no/total no (\%) & $95 / 578(16.4)$ & $49 / 268(18.3)$ & $46 / 310(14.8)$ & 0.265 \\
\hline Myocarditis, patient no/total no (\%) & $30 / 578(5.2)$ & $7 / 268(2.6)$ & $23 / 310(7.4)$ & 0.009 \\
\hline Valvulitis, patient no/total no (\%) & $178 / 578(30.8)$ & $100 / 268(37.3)$ & $78 / 310(25.2)$ & 0.002 \\
\hline Pericarditis, patient no/total no (\%) & $44 / 578(7.6)$ & $23 / 268(8.6)$ & $21 / 310(6.8)$ & 0.414 \\
\hline Coronary artery dilatation, patient no/total no (\%) & $57 / 578(9.9)$ & $36 / 268(13.4)$ & $21 / 310(6.8)$ & 0.007 \\
\hline Coronary artery aneurysm, patient no/total no (\%) & $5 / 578(0.9)$ & $4 / 268(1.5)$ & $1 / 310(0.3)$ & 0.188 \\
\hline \multicolumn{5}{|l|}{ Pharmacotherapy } \\
\hline Intravenous immunoglobulin (IVIG), $n(\%)$ & $571(93)$ & $269(97.1)$ & $302(89.6)$ & $<0.001$ \\
\hline Corticosteroids, $n(\%)$ & $514(83.8)$ & $240(87)$ & $274(81.3)$ & 0.059 \\
\hline Acetylsalicylic acid, $n(\%)$ & 409 (66.6) & $229(82.7)$ & $180(53.4)$ & $<0.001$ \\
\hline Anticoagulants, $n(\%)$ & $368(59.9)$ & $165(59.6)$ & $203(60.2)$ & 0.866 \\
\hline Oxygen replacement, $n(\%)$ & $161(26.2)$ & $73(26.4)$ & $88(26.1)$ & 0.946 \\
\hline Inotropes, $n(\%)$ & $117(19.1)$ & $42(15.2)$ & $75(22.3)$ & 0.026 \\
\hline Immunomodulatory therapy ${ }^{\mathrm{m}}, n(\%)$ & $39(6.4)$ & $21(7.6)$ & $18(5.3)$ & 0.257 \\
\hline Plasma exchange, $n(\%)$ & $10(1.6)$ & $6(2.2)$ & $4(1.2)$ & 0.359 \\
\hline \multicolumn{5}{|l|}{ Outcomes } \\
\hline $\mathrm{NIMV}^{\mathrm{n}}, n(\%)$ & $59(9.6)$ & $21(7.6)$ & $38(11.3)$ & 0.122 \\
\hline Intubation, $n(\%)$ & $24(3.9)$ & $10(3.6)$ & $14(4.2)$ & 0.729 \\
\hline Prone position, $n(\%)$ & $5(0.8)$ & $1(0.4)$ & $4(1.2)$ & 0.385 \\
\hline Extracorporeal membrane oxygenation, $n(\%)$ & $4(0.7)$ & $1(0.4)$ & $3(0.9)$ & 0.631 \\
\hline Development of bradycardia, $n(\%)$ & $64(10.4)$ & $34(12.3)$ & $30(8.9)$ & 0.174 \\
\hline Death, $n(\%)$ & $11(1.8)$ & $6(2.2)$ & $5(1.5)$ & 0.556 \\
\hline
\end{tabular}

${ }^{a}$ American Heart Association criteria for the definition of KD is to have persistent fever and 4 of the following 5 mucocutaneous features: erythema and cracking of lips/strawberry tongue and/or erythema of oral and pharyngeal mucosa, bilateral bulbar conjunctival injection without exudate, rash, erythema and edema of the hands and feet in acute phase and/or periungual desquamation in subacute phase, and cervical lymphadenopathy

${ }^{\mathrm{b}} \mathrm{ICU}$ Intensive care unit

${ }^{\mathrm{c}} \mathrm{IQR}$ interquartile range

${ }^{\mathrm{d}}$ Respiratory failure was defined as tachypnea (age-appropriate), respiratory distress and/or need for oxygen treatment, and/or clinical signs of pneumonia and /or ARDS and/or pulmonary embolism 
Table 3 (continued)

${ }^{\mathrm{e}}$ Hepatik failure was defined elevated bilirubin or elevated liver enzymes

${ }^{\mathrm{f}}$ Acute kidney injury defined by creatinine level greater than the upper limit for age

${ }^{\mathrm{g}}$ Hematologic failure was defined as d-Dimer elevation and/or neutrophilia and/or lymphopenia and/or thrombocytopenia. Neutrophilia was defined as ANC $\geq 7700 / \mu \mathrm{g} / \mathrm{L}$. Lymphopenia was defined as below $4500 \mu$ Lin children under 8 months of age and below $1500 \mu \mathrm{L}$ above eight months of age

${ }^{\text {h}}$ Ravelli criteria for risk assessment of secondary macrophage activation syndrome was used

${ }^{\mathrm{i}}$ Gastrointestinal involvement was defined as symptoms that related with gastrointestinal system such as (abdominal pain and/or vomiting and/or diarrhea) and/or elevated liver enzymes and/or elevation in serum lipase or amylase to three times or greater than the upper limit of normal

${ }^{\mathrm{j} N e u r o l o g i c a l ~ i n v o l v e m e n t ~ w a s ~ d e f i n e d ~ a s ~ e n c e p h a l o p a t h y, ~ a s e p t i c ~ m e n i n g i t i s, ~ o r ~ c e r e b r o v a s c u l a r ~ d i s e a s e ~}$

${ }^{\mathrm{k}}$ Cardiovadscular involvement was defined as shock and/or elevated troponin and/or elevated BNP and/or elevated Nt-ProBNP and/or abnormal echocardiogram and/or arrhythmia

${ }^{1}$ Shock was defined as needing inotrope support or fluid resuscitation $>20 \mathrm{~mL} / \mathrm{kg}$

${ }^{\mathrm{m}}$ Immunomodulatory therapy includes using of anakinra, tocilizumab, and infliximab. Anakinra was administered to 31 patients, tocilizumab to 8 patients, and infliximab to 1 patient

${ }^{\mathrm{n}} N I M V$ non-invasive mechanical ventilation

patients with coronary artery aneurysms had KD-like features. The frequency of coronary artery aneurysms was not statistically significant between the two groups $(p=0.188)$. We suspected that the small number of patients with coronary artery aneurysms caused this situation. Also, valvulitis was found significantly more frequently in the group whose characteristics overlapped with KD. A significantly higher rate of myocarditis was found in the other group.

High acute phase responses were observed in our study, especially higher procalcitonin levels. In patients whose

Table 4 Univariate logistic regression analysis is shown independent predictors for overlapping with Kawasaki disease

\begin{tabular}{|c|c|c|c|c|c|}
\hline & \multirow[t]{2}{*}{$\operatorname{Exp}(\beta)$} & \multicolumn{2}{|c|}{$\begin{array}{l}95 \% \text { C.I. for } \\
\exp (\beta)\end{array}$} & \multirow[t]{2}{*}{$p$ value } & \multirow[t]{2}{*}{ Wald Statistics } \\
\hline & & Lower & $\overline{\text { Upper }}$ & & \\
\hline \multicolumn{6}{|l|}{ Age groups } \\
\hline 5-12 & Ref & - & - & - & - \\
\hline $0-5$ & 0.641 & 0.434 & 0.948 & 0.026 & 4.967 \\
\hline$>12$ & 0.330 & 0.211 & 0.514 & $<0.001$ & 23.985 \\
\hline Nausea & 0.708 & 0.504 & 0.994 & 0.046 & 3.970 \\
\hline Fatigue & 1.708 & 1.069 & 2.730 & 0.025 & 5.005 \\
\hline Lethargy & 3.299 & 1.647 & 6.611 & 0.001 & 11.335 \\
\hline $\begin{array}{l}\text { Lymphocyte, } \\
\text { mm }^{3}\end{array}$ & 1.000 & 1.000 & 1.000 & 0.155 & 2.019 \\
\hline $\begin{array}{l}\text { Neutrophil, } \\
\text { mm}^{3}\end{array}$ & 1.000 & 1.000 & 1.000 & 0.022 & 5.262 \\
\hline $\begin{array}{l}\text { Hemoglobin, } \\
\text { g/dl }\end{array}$ & 0.937 & 0.847 & 1.038 & 0.215 & 1.538 \\
\hline $\begin{array}{l}\text { Thrombocyte, } \\
\text { mm }^{3}\end{array}$ & 1.000 & 1.000 & 1.000 & $<0.001$ & 15.528 \\
\hline ALT, $\mu / \mathbf{L}$ & 1.002 & 1.000 & 1.005 & 0.094 & 2.801 \\
\hline Ferritin, $\mu \mathrm{g} / \mathrm{L}$ & 1.000 & 1.000 & 1.000 & 0.069 & 3.308 \\
\hline D-dimer, $\mu \mathrm{g} / \mathrm{L}$ & 1.000 & 1.000 & 1.000 & 0.053 & 3.760 \\
\hline Albumin, g/dL & 0.445 & 0.339 & 0.585 & $<0.001$ & 33.774 \\
\hline
\end{tabular}

clinical features overlapped with KD, procalcitonin levels were almost 15 times higher than normal, and even in patients without overlap with $\mathrm{KD}$, levels were 8 times higher than normal. A study by Bar-Meir from Israel has examined 10 patients with MIS-C and 5 patients who met both the MIS-C and KD definitions [20]. They have found that lymphopenia was an independent predictor of MIS-C (mean lymphocyte count $700 \times \mu \mathrm{L}$ at MIS-C, $1500 \times \mu \mathrm{L}$ at MISC and KD criteria; odds ratio of $24,95 \%$ CI $1.3-326$, $p=0.02$ ). Similar to this result, lymphocyte counts were low in patients whose clinical manifestations overlapped with or without KD, but significantly lower in those whose features overlapped with KD (1080 vs. $1280 \mu \mathrm{L}, p=0.028)$.

Table 5 Multivariate logistic regression analysis is shows independent predictors of overlapping with Kawasaki disease

\begin{tabular}{|c|c|c|c|c|c|}
\hline & \multirow[t]{2}{*}{$\operatorname{Exp}(\beta)$} & \multicolumn{2}{|c|}{$\begin{array}{l}95 \% \text { CI for } \\
\exp (\beta)\end{array}$} & \multirow[t]{2}{*}{$p$ value } & \multirow[t]{2}{*}{ Wald statistics } \\
\hline & & Lower & Upper & & \\
\hline \multicolumn{6}{|l|}{ Age groups } \\
\hline $5-12$ & Ref & & & & 20.913 \\
\hline $0-5$ & 0.883 & 0.574 & 1.359 & 0.571 & 0.321 \\
\hline$>12$ & 0.345 & 0.217 & 0.550 & $<0.001$ & 19.985 \\
\hline Lethargy & 2.601 & 1.244 & 5.439 & 0.011 & 6.449 \\
\hline $\begin{array}{l}\text { Hemoglobin, } \\
\text { g/dl }\end{array}$ & 1.150 & 1.011 & 1.307 & 0.033 & 4.544 \\
\hline $\begin{array}{l}\text { Neutrophil } \\
\text { count }\end{array}$ & 1.000 & 1.000 & 1.000 & 0.018 & 5.636 \\
\hline Albumin, $\mathbf{g} / \mathbf{d L}$ & 0.408 & 0.298 & 0.559 & $<0.001$ & 31.074 \\
\hline
\end{tabular}

Variables from the univariate logistic regression analysis with a statistical result of $p<0.250$ were included in the multivariate logistic regression analysis. Age groups, sex, nausea, fatigue, lethargy, lymphocyte count, neutrophil count, hemoglobin level, platelet count, ferritin level, D-dimer level, albumin level, and ALT results were evaluated in the analysis 
However, no significant an association was found in the multilogistic regression analysis. In the multilogistic regression analysis, we found that age $>12$ years reduced the risk of overlap with KD by $66 \%$, lethargy increased the risk of overlap with KD by 2.6 -fold in MIS-C patients. The laboratory results showed that each unit increase in albumin decreased the probability of overlap with Kawasaki disease by $60 \%$.

Transient bradycardia was noted in approximately $10 \%$ of our patients after initiation of treatment. Two of these patients required follow-up in the intensive care unit. Bradycardia could be related to edema formation in cardiomyocytes and its effects on the cardiac excitation conduction system. In addition, the bradycardia was observed two days after the start of treatment, and the use of intensive steroids and IVIG in these patients suggests that it could also be treatment-related side effects. Moreover, long-term followup of patients with cardiac magnetic resonance imaging and advanced echocardiographic procedures could be performed to improve the understanding of the cardiac effects of MIS-C.

Almost $30 \%$ of the patients required a stay in the intensive care unit. This is lower than in the Feldstein cohort, in which $80 \%$ of patients received intensive care; in the Godfred-Cato cohort, $63 \%$ of the patients received intensive care $[6,9]$. The need for ICU stays and the development of the shock were statistically higher in patients with characteristics that did not overlap with KD. It was hypothesized that the patients with clinical findings consistent with $\mathrm{KD}$ (mucosal changes, bulbar conjunctivitis, lymphadenopathy, and rash) would be more easily recognized and treated by experts, whereas the patients with high fever without these findings required a longer diagnose period which leading to a later start of treatment.

In a recent MISC data from CDC, $0.8 \%$ of the 4196 identified MIS-C patients died. In our study, the mortality rate was $1.7 \%$, similar to a study by Whittacker et al. [4]. Five of the deceased patients had comorbidities; two of them had acute lymphoblastic leukemia, one had aplastic anemia, one had ataxia telangiectasia, and one had congenital cytomegalovirus disease. In addition, there were signs of incomplete KD in 6 deceased patients, and a sign of complete $\mathrm{KD}$ in one deceased patient.

It is noteworthy that few reports of MIS-C have been reported in Asian countries where the COVID-19 pandemic began and the incidence of $\mathrm{KD}$ is highest [7, 8]. Understanding the pathophysiology of this emerging disease may provide welcome insights into our understanding of KD. Although not the same disease, MIS-C and KD may be sibling diseases that fall under a larger syndrome of post-acute autoimmune febrile responses to infections.

Our study has limitations. First, there was no consensus on treatment methods for patients. Although long-term followup of patients is not the primary purpose of this case series, studying the long-term outcomes of MISC patients with and without overlap with KD may be important to improve the understanding of both KD and MIS-C outcomes.
In conclusion, in our case series, the rate of MIS-C patients with clinical features overlapping with Kawasaki disease was higher than in the literature. Median age was lower compared with patients without overlap with Kawasaki disease. In addition, lymphocyte and platelet counts were lower and ferritin and procalcitonin levels were significantly elevated in patients with overlap with Kawasaki disease. Pediatricians should be aware of MIS-C, which has similarities to KD but also has its own characteristics. It should also be remembered that patients may develop complications such as bradycardia during hospitalization, and careful follow-up programs for this condition are important in terms of long-term outcomes.

Authors' contributions Drs Yilmaz-Ciftdogan, Ekemen-Keles, Turel, Dinleyici, Akturk, Torun, Ciftci, Karbuz, and Kara conceptualized and designed the study, collected data, drafted the initial manuscript, and reviewed and revised the manuscript. Drs Cetin, Turel, Sert, Sari, Orbak, Oguz Sahin, Varan, Akturk, Durak, Oflaz, Kara, MD, Karpuz, Petmezci, Hatipoglu, Oncel, Turgut, Elmali, Assoc Prof, Ayper Somer, MD, Prof, Necdet Kuyucu, MD, Prof, Ener Cagri Dinleyici, MD, Prof, Zafer Kurugöl, MD, Prof, Ergin Ciftci, MD, Prof, Ates Kara, MD, Prof Karabulut, Emiroglu, Bagci, Buyukcam, Erdeniz Arga, Yesil, Cakıcı, Karbuz, Sahbudak Bal, Kara, Ozer, Metin Akcan, Elmas Bozdemir, Anil, Uygun, Kilic, Hancerli Torun, Umit, Sutcu, Ozmen, Mr Elmali, Dinleyici, and Çiftci designed the data collection instruments, collected data, critically reviewed the manuscript for important intellectual content, and revised the manuscript. Drs Asrak, Kara Aksay, Ugur, Birbilen, Duramaz, Ozkan, Burakay, Yildirim Arslan, MD, Karadag Oncel, Celik, Kilic, Ozen, Sarikaya, Demirkol, and Arslan conceptualized and designed the study, collected data drafted the initial manuscript, reviewed, coordinated and supervised data collection, and critically reviewed the manuscript for important intellectual content.

Availability of data and material All data and document information is available to give to the European Journal of Pediatrics.

\section{Declarations}

Ethics approval Ethics committee approval was obtained.

Consent to participate All authors approved the final manuscript as submitted and agree to be accountable for all aspects of the work.

Consent for publication All authors have given consent for the study to be published in European Journal of Pediatrics journal.

Conflict of interests The authors declare no competing interests.

\section{References}

1. Dong Y, Mo X, Hu Y, Qi X, Jiang F, Jiang Z, Tong S (2020) Epidemiological characteristics of 2143 pediatric patients with 2019 coronavirus disease in China. Pediatrics 145(6):e20200702. https://doi.org/10.1542/peds.2020-0702

2. Riphagen S, Gomez X, Gonzalez-Martinez C, Wilkinson N, Theocharis P (2020) Hyperinflammatory shock in children during 
COVID-19 pandemic. Lancet 395:1607-1608. https://doi.org/10. 1016/S0140-6736(20)31094-1

3. Verdoni L, Mazza A, Gervasoni A, Martelli L, Ruggeri M, Ciuffreda M, Bonanomi E, D’Antiga L (2020) An outbreak of severe Kawasakilike disease at the Italian epicentre of the SARS-CoV-2 epidemic: an observational cohort study. Lancet 395:1771-1778. https://doi.org/ 10.1016/S0140-6736(20)31103-X

4. Whittacker E, Bamford A, Kenny J et al (2020) Clinical characteristics of 58 children with a pediatric inflammatory multisystem syndrome temporally associated with SARS-CoV-2. JAMA 324:259-269. https://doi.org/10.1001/jama.2020.10369

5. Dufort EM, Koumans EH, Chow EJ et al (2020) Multisystem inflammatory syndrome in children in New York State. N Engl J Med 383:347-358. https://doi.org/10.1056/NEJMoa2021756

6. Feldstein LR, Rose EB, Horwitz SM et al (2020) Multisystem inflammatory syndrome in U.S. children and adolescents. N Engl J Med 383:334-346. https://doi.org/10.1056/NEJMoa2021680

7. Fukuda S, Kaneta M, Miyake M, Ohya T, Miyakawa K, Iwamoto M, Ito S (2021) A case of multisystem inflammatory syndrome in children in a Japanese boy: with discussion of cytokine profile. Mod Rheumatol Case Rep 21(7):1-6. https://doi.org/10.1080/24725625.2021.1920140

8. Jain S, Sen S, Lakshmivenkateshiah S (2020) Multisystem inflammatory syndrome in children with COVID-19 in Mumbai, India. Indian Pediatr 57:1015-1019. https://doi.org/10.1007/ s13312-020-2026-0

9. Godfred-Cato S, Bryant B, Leung J et al (2020) COVID-19-associated multisystem inflammatory syndrome in children - United States, March-July 2020. MMWR Morb Mortal Wkly Rep 69:1074-1080. https://doi.org/10.15585/mmwr.mm6932e2externalicon.

10. McCrindle BW, Rowley AH, Newburger JW et al (2017) Diagnosis, treatment, and long-term management of Kawasaki disease: a scientific statement for health professionals from the American Heart Association. Circulation 135(17):e927-e999. https://doi. org/10.1161/CIR.0000000000000484

11. Makino N, Nakamura Y, Yashiro M et al (2015) Descriptive epidemiology of Kawasaki disease in Japan, 2011-2012: from the results of the 22nd nationwide survey. J Epidemiol 25:239. https:// doi.org/10.2188/jea.JE20140089

12. Kim YJ, Park H, Choi YY et al (2020) Defining association between COVID-19 and the multisystem inflammatory syndrome in children through the pandemic. J Korean Med Sci 35:e204. https://doi.org/10.3346/jkms.2020.35.e204

13. Centers for Disease Control and Prevention (2021) Reported cases of multisystem inflammatory syndrome in children (MISC) in the United States https://www.cdc.gov/mis/cases/index.html. Accessed Jun 2021

14. Grimaud M, Starck J, Levy M, Marais C et al (2020) Acute myocarditis and multisystem inflammatory emerging disease following SARS-CoV-2 infection in critically ill children. Ann Intensive Care 10(1):69. https://doi.org/10.1186/s13613-020-00690-8

15. Cao L, Zhang S, Luo X et al (2020) Myocardium injury biomarkers predict prognosis of critically ill coronavirus disease 2019 (COVID-19) patients. Ann Palliat Med 9(6):4156-4165. https:// doi.org/10.21037/apm-20-2112

16. Centers for Disease Control and Prevention (2020) Multisystem inflammatory syndrome in children (MIS-C) associated with coronavirus disease 2019 (COVID-19). https://www.cdc.gov/mmwr/ volumes $/ 69 / \mathrm{wr} / \mathrm{mm} 6932 \mathrm{e} 2 . \mathrm{htm}$. Accessed Aug 2020

17. World Health Organization (2020) Multisystem inflammatory syndrome in children and adolescents with COVID-19. https:// www.who.int/news-room/commentaries/detail/multisysteminflammatory-syndrome-in-children-and-adolescents-withcovid-19. Accessed May 2020

18. Centers for Disease Control and Prevention (2021) Health department-reported cases of multisystem inflammatory syndrome in children (MIS-C) in the United States. https://www.cdc.gov/mis/ cases/index.html. Accessed 30 Jun 2021

19. Jhaveri S, Ahluwalia N, Kaushik S et al (2021) Longitudinal echocardiographic assessment of coronary arteries and left ventricular function following multisystem inflammatory syndrome in children. J Pediatr 228:290-293.e1. https://doi.org/10.1016/j.jpeds. 2020.08.002

20. Bar-Meir M, Guri A, Godfrey ME et al (2021) Characterizing the differences between multisystem inflammatory syndrome in children and Kawasaki disease. Sci Rep 5;11(1):13840. https:// doi.org/10.1038/s41598-021-93389-0

Publisher's Note Springer Nature remains neutral with regard to jurisdictional claims in published maps and institutional affiliations.

\section{Authors and Affiliations}

Dilek Yilmaz Ciftdogan ${ }^{1,2} \cdot$ Yildiz Ekemen Keles $^{2}$ - Benhur Sirvan Cetin ${ }^{3} \cdot$ Nazan Dalgic Karabulut $^{4}$. Melike Emiroglu ${ }^{5}$ Zafer Bagci ${ }^{6}$ Ayse Buyukcam ${ }^{7}$ Emine Hafize Erdeniz ${ }^{8}$. Gul Arga ${ }^{9}$. Edanur Yesil ${ }^{10}$. Ozlem Cakici ${ }^{11}$. Adem Karbuz ${ }^{12}$. Zumrut Sahbudak Bal ${ }^{13}$. Soner Sertan Kara ${ }^{14}$. Arife Ozer ${ }^{15}$. Ozge Metin Akcan ${ }^{16}$. Sefika Elmas Bozdemir ${ }^{17}$. Ayse Berna Anil ${ }^{1}$ Hatice Uygun ${ }^{18}$. Omer Kilic ${ }^{19}$. Selda Hancerli Torun ${ }^{20}$. Zuhal Umit $^{21}$. Murat Sutcu ${ }^{22}$. Berfin Ozgokce Ozmen ${ }^{23}$. Hatice Karaoglu Asrak ${ }^{24}$. Gulsum Alkan ${ }^{5}$. Ahu Kara Aksay ${ }^{2}$. Cuneyt Ugur ${ }^{6}$. Ahmet Ziya Birbilen ${ }^{7}$. Burcu Bursal Duramaz ${ }^{25}$. Esra Akyuz Ozkan ${ }^{8}$. Ozgur Burakay ${ }^{11}$. Sema Yildirim Arslan ${ }^{13} \cdot$ Eda Karadag Oncel $^{2} \cdot$ Serkan Fazli Celik ${ }^{14} \cdot$ Ahmet Osman Kilic $^{16} \cdot$ Seval Ozen $^{18}$. Remzi Sarikaya ${ }^{15}$. Demet Demirkol ${ }^{20}$. Gazi Arslan ${ }^{24}$. Ozden Turel ${ }^{25} \cdot$ Ahmet Sert $^{5} \cdot$ Ergul Sari $^{26} \cdot$ Zerrin Orbak $^{27}$. Irfan Oguz Sahin ${ }^{8}$. Celal Varan ${ }^{18}$. Hacer Akturk ${ }^{28}$. Sadiye Kubra Tuter Oz ${ }^{5}$. Fatih Durak ${ }^{7}$. Mehmet Burhan Oflaz ${ }^{16}$. Manolya Kara ${ }^{22}$. Derya Karpuz ${ }^{23}$. Mey Talip Petmezci ${ }^{12}$. Nevin Hatipoglu ${ }^{26}$. Selim Oncel ${ }^{11} \cdot$ Mehmet Turgut $^{18}$. Ferhan Elmali ${ }^{1}$ - Ayper Somer ${ }^{20} \cdot$ Necdet Kuyucu $^{23} \cdot$ Ener Cagri Dinleyici $^{19} \cdot$ Zafer Kurugöl $^{13} \cdot$ Ergin Ciftci $^{9}$. Ates Kara ${ }^{29}$

Yildiz Ekemen Keles kutupylz@hotmail.com
Dilek Yilmaz Ciftdogan drdilekyilmaz@hotmail.com 
Benhur Sirvan Cetin

benhurcetin@erciyes.edu.tr

Nazan Dalgic Karabulut

nazandalgic@ttmail.com

Melike Emiroglu

mkeser17@gmail.com

Zafer Bagci

zfrbagci@gmail.com

Ayse Buyukcam

dr.aysebaktir@gmail.com

Emine Hafize Erdeniz

dregemine5658@hotmail.com

Gul Arga

gul.tepebasi@gmail.com

Edanur Yesil

eda_hacettepe@hotmail.com

Ozlem Cakici

zlmckc@gmail.com

Adem Karbuz

karbuzadem@hotmail.com

Zumrut Sahbudak Bal

z.sahbudak@gmail.com

Soner Sertan Kara

drsoner@yahoo.com

Arife Ozer

drozerarife@gmail.com

Ozge Metin Akcan

drozgemetin@gmail.com

Sefika Elmas Bozdemir

drsefika@hotmail.com

Ayse Berna Anil

aysebernaanil@hotmail.com

Hatice Uygun

ozhanhatice@hotmail.com

Omer Kilic

omerkilic7@yahoo.com

Selda Hancerli Torun

seldahancerli@hotmail.com

Zuhal Umit

zuhalumit7@gmail.com

Murat Sutcu

sutcu13@yahoo.com

Berfin Ozgokce Ozmen

dr.b.ozmen@hotmail.com

Hatice Karaoglu Asrak

hatice_karaoglu@msn.com

Gulsum Alkan

galkan-85@hotmail.com

Ahu Kara Aksay

ahukara01@hotmail.com

Cuneyt Ugur

cugur70@gmail.com
Ahmet Ziya Birbilen

abirbilen@hotmail.com

Burcu Bursal Duramaz

burcubursal@hotmail.com

Esra Akyuz Ozkan

uzdresra@gmail.com

Ozgur Burakay

ozgursancar@hotmail.com

Sema Yildirim Arslan

semayildirimarslan@gmail.com

Eda Karadag Oncel

dredakaradag@gmail.com

Serkan Fazli Celik

docser2003@yahoo.com

Ahmet Osman Kilic

drahmetosmankilic@gmail.com

Seval Ozen

drsevalcevik@hotmail.com

Remzi Sarikaya

drremzisarikaya@gmail.com

Demet Demirkol

d-demirkol@hotmail.com

Gazi Arslan

gaziarslan@gmail.com

Ozden Turel

barisbulent98@yahoo.com

Ahmet Sert

ahmetsert2@hotmail.com

Ergul Sari

drergulsari@gmail.com

Zerrin Orbak

zerrinorbak@yahoo.com

Irfan Oguz Sahin

rfnshn@yahoo.com

Celal Varan

celalvaran@hotmail.com

Hacer Akturk

hacergunakturk@gmail.com

Sadiye Kubra Tuter Oz

sadiyettr@hotmail.com

Fatih Durak

fatihdurak44@hotmail.com

Mehmet Burhan Oflaz

mburhanoflaz@gmail.com

Manolya Kara

manolya_kara@yahoo.com

Derya Karpuz

drderyakarpuz@gmail.com

Mey Talip Petmezci

meytalip@gmail.com

Nevin Hatipoglu

naydin9@ mynet.com 
Selim Oncel

selim.oncel@kocaeli.edu.tr

Mehmet Turgut

drmehmetturgut@yahoo.com

Ferhan Elmali

elmaliferhan@yahoo.com

Ayper Somer

ayper.somer@gmail.com

Necdet Kuyucu

nkuyucu@yahoo.com

Ener Cagri Dinleyici

enercagri@gmail.com

Zafer Kurugöl

zkurugol@gmail.com

Ergin Ciftci

erginciftci@gmail.com

Ates Kara

ateskara@hacettepe.edu.tr

Izmir Katip Celebi University, Izmir, Turkey

2 Health Sciences University Tepecik Training and Research Hospital, Izmir, Turkey

3 Erciyes University Hospital, Kayseri, Turkey

4 Health Sciences University Sisli Hamidiye Etfal Training and Research Hospital, Istanbul, Turkey

5 Selcuk University Hospital, Konya, Turkey

6 University of Health Sciences Konya Health Application and Research Center, Konya, Turkey

7 Gaziantep CG Obstetrics and Children's Hospital, Gaziantep, Turkey
10

11

12 Istanbul Professor Doctor Cemil Tascioglu City Hospital, Istanbul, Turkey

13 Ege University Hospital, Izmir, Turkey

14 Aydin Adnan Menderes University, Aydin, Turkey

15 Van Training and Research Hospital, Van, Turkey

16 Necmettin Erbakan University, Meram Hospital, Konya, Turkey

17 Bursa Dortcelik Children's Hospital, Bursa, Turkey

18 Adiyaman University Hospital, Adiyaman, Turkey

19 Osmangazi University Hospital, Eskisehir, Turkey

20 Istanbul University Hospital, Istanbul, Turkey

21 Manisa City Hospital, Manisa, Turkey

22 Istinye University Hospital, Istanbul, Turkey

23 Mersin University Hospital, Mersin, Turkey

24

25

26

27

28

29

Dokuz Eylul University Hospital, İzmir, Turkey

Bezmialem Vakif University Hospital, Istanbul, Turkey

Bakirkoy Sadi Konuk Children Hospital, Istanbul, Turkey

Ataturk University Hospital, Erzurum, Turkey

Koc University Hospital, Istanbul, Turkey

Hacettepe University Hospital, Ankara, Turkey 